\title{
PARTICLE TRAJECTORIES AND THE PERCEPTION OF CLASSICAL MOTION IN THE FREE PROPAGATION OF WAVE PACKETS.
}

\author{
John Briggs ${ }^{1}$ \\ ${ }^{1}$ University of Freiburg
}

October 25, 2021

\begin{abstract}
The free propagation in time of a normalisable wave packet is the oldest problem of continuum quantum mechanics. Its motion from microscopic to macroscopic distance is the way in which most quantum systems are detected experimentally. Although much studied and analysed since 1927 and presented in many text books, here the problem is re-appraised from the standpoint of semi-classical mechanics. Particular aspects are the emergence of deterministic trajectories of particles emanating from a region of atomic dimensions and the interpretation of the wave function as describing a single particle or an ensemble of identical particles. Of possible wave packets, that of gaussian form is most studied due to the simple exact form of the time-dependent solution in real and in momentum space. Furthermore, this form is important in laser optics. Here the equivalence of the time-dependent Schroedinger equation to the paraxial equation for the propagation of light is demonstrated explicitly. This parallel helps to understand the relevance of trajectory concepts and the conditions necessary for the perception of motion as classical.
\end{abstract}

\section{Hosted file}

Version 2021.pdf available at https://authorea.com/users/442552/articles/542789-particletrajectories-and-the-perception-of-classical-motion-in-the-free-propagation-of-wavepackets 